\title{
Adiponectin oligomers are similarly distributed in adequate-for-gestational-age obese children irrespective of feeding in their first year
}

\author{
Flavia Prodam ${ }^{1-3}$, Marta Roccio ${ }^{1,4}$, Letizia Trovato5, Roberta Ricotti' ${ }^{1}$ Stefania Moia', Enza Giglione ${ }^{1}$, Antonella Petri ${ }^{1,3}$, \\ Gillian E. Walker', Simonetta Bellone ${ }^{1,3}$ and Gianni Bona ${ }^{1,3,4}$
}

BACKGROUND: Nutrition and growth in early postnatal life have a role in future diseases. Our aim was to investigate adiponectin oligomers in adequate-for-gestational-age obese children with respect to type and duration of feeding in the first year of life.

METHODS: Adiponectin oligomers and cardiometabolic risk factors were measured in 113 adequate-for-gestational-age obese children, divided into group A (prolonged breast feeding, $>6 \mathrm{mo}$ ), group $B$ (short breast feeding, 1-6 mo), and group C (formula feeding from birth).

RESULTS: All the parameters were similar among the groups. Adiponectin oligomers did not correlate with gestational age, months of breast feeding, and time of weaning. Total and high-molecular weight adiponectin were differently distributed across gender and pubertal stages $(P<0.02)$, being lower in males from the start of puberty. Prepregnancy BMI and at the end of the pregnancy were negatively associated $(P<$ 0.04 ) with total and medium-molecular weight adiponectin in female and male offspring, respectively.

CONCLUSIONS: Adiponectin oligomers and metabolic characteristics are similarly distributed in adequate-for-gestationalage obese children, irrespective of the type and duration of the feeding in the first year of life. Gender and mother's BMI in pregnancy are contributors to adiponectin regulation. Further studies will explain whether breastfeeding protects against metabolic impairment later in life.

N utrition and growth in early postnatal life have been indicated to have a central role in disease predisposition later in life, including obesity, type 2 diabetes, and cardiovascular diseases. This concept is the core of nutritional programing, which indicates that both prenatal and early postnatal life constitute critical windows for future health by introducing a complex perspective on the pathogenesis of metabolic alterations (1-3). The contribution of breastfeeding is one of the most investigated topics with the European Society of Pediatric Gastroenterology
Hepatology and Nutrition (ESPGHAN) recommendations being to maintain exclusively breastfeeding for at least 6 mo (4). Although several studies have shown that breastfeeding protects from obesity, dyslipidemia, hypertension, and type 2 diabetes, some data are controversial $(3,5,6)$. While the mechanisms involved in reducing the risk of future diseases need to be further explored, it has been suggested that hormones and neuropeptides play a crucial role. Amongst these, adiponectin seems to be age-dependently regulated in infants born small for gestational age (SGA), and the secretion in blood could also be dependent on breastfeeding or formula feeding $(7,8)$. With respect to other adipokines, adiponectin has insulin-sensitizing effects, regulates centrally food intake and body weight, and possesses anti-inflammatory properties, demonstrating a clinical relevance in obesity-associated complications $(9,10)$. Its synthesis and secretion are specific to adipocytes (11). Circulating adiponectin is negatively correlated to BMI and it decreases further in subjects with insulin resistance, type 2 diabetes, and cardiovascular diseases (12). Adiponectin is found in the circulation as three multimeric forms: trimer (LMW, low molecular weight), hexamer (MMW, medium molecular weight), and multiple monomers (12-18 monomers, HMW, high molecular weight). The HMW form is considered the key bioactive form, particularly with respect to insulin action $(9,10,13)$. It has also been demonstrated that adiponectin concentrations in cord blood are higher than those reported in pediatric and adult subjects $(14,15)$. Interestingly, adiponectin levels in human milk are higher than those in leptin and are almost entirely comprised of the HMW form (16). Whether adiponectin is inversely associated with adiposity is unknown, as it is unclear if adiponectin levels in obese children born adequate for gestational age (AGA) could be also influenced by the type of milk used and/or the duration of feeding in the first year of life.

As such, the first aim of this study was to investigate total, HMW, and MMW adiponectin concentrations in AGA obese children with respect to breastfeeding or formula feeding in the first year of life and with respect to the duration of breastfeeding.

\footnotetext{
'Division of Pediatrics, Department of Health Sciences, University of Piemonte Orientale, Novara, Italy; ${ }^{2}$ Endocrinology, Department of Clinical \& Experimental Medicine, University of Piemonte Orientale, Novara, Italy; ${ }^{3}$ Interdisciplinary Center for Obesity Study (ICOS), University of Piemonte Orientale, Novara, Italy; ${ }^{4}$ nterdisciplinary Research Center of Autoimmune Diseases (IRCAD), University of Piemonte Orientale, Novara, Italy; ${ }^{5}$ Division of Endocrinology, Diabetology and Metabolism, Department of Medical Sciences, University of Torino, Torino, Italy. Correspondence: Flavia Prodam (flavia.prodam@med.unipmn.it) 
The second aim was to investigate whether these subgroups present a different obese phenotype in terms of comorbidities.

\section{RESULTS}

\section{Clinical and Biochemical Characteristics}

A total of 120 subjects were recruited to meet the inclusion criteria. Seven of them were subsequently excluded because at the control of anamnestic data, breastfeeding in the first month was not exclusive.

The complete anthropometrical and biochemical fasting data are summarized in Table 1. Groups A, B, and C were similar in terms of anthropometric and metabolic parameters. Group B subjects were moderately older $(P<0.05)$ than the other two groups. Group C subjects presented a slightly lower birth weight than group A subjects $(P<0.03)$. Mothers of groups A and $\mathrm{B}$ children had a similar prepregnancy weight and BMI, and at the end of pregnancy. Mothers of group $\mathrm{C}$ children had a higher weight and $\mathrm{BMI}$ at the end of pregnancy with respect to groups $\mathrm{A}$ and $\mathrm{B}(P<0.02)$, and also a higher prepregnancy weight with respect to group $\mathrm{A}(P<0.03)$.

The crude means of total, HMW, and MMW adiponectin were similar among the three groups. The weighted means (models 1, 2 and 3) of total, HMW, and MMW adiponectin remained similar among the groups. The HMW adiponectin levels were differently distributed across gender and pubertal stages $(P<0.02)$, with lower levels observed in males from the start of puberty (Figure 1).

\section{Correlations}

Neither total adiponectin nor HMW and MMW adiponectin correlated with gestational age, weight and length at birth, months of breastfeeding, and time of weaning, in both sexes. The total and HMW adiponectin correlated with BMI, body mass index standard deviation scores (BMISDS), systolic blood pressure (SBP), high-density lipoprotein (HDL)-cholesterol, and glucose during an oral glucose tolerance test (OGTT) in males, but not in females. Total and HMW adiponectin also correlated with insulin levels during OGTT and diastolic blood pressure (DBP), respectively, in both sexes (Table 2). The correlations of total and HMW adiponectin were maintained when weighted for covariates. MMW adiponectin did not correlate with any of the selected variables for the children. Moreover, the total adiponectin levels in female offspring were negatively correlated with the maternal prepregnancy and at the end of pregnancy BMI $(P<0.05)$. On the contrary, MMW adiponectin in male offspring negatively correlated with maternal prepregnancy $(r:-0.288, P<0.04)$ and at the end of pregnancy BMI $(r:-0.309, P<0.02)$. These correlations were not modified by the type of feeding in the first year of life.

\section{DISCUSSION}

Previous studies have evaluated HMW adiponectin levels in children born SGA in the first 4 and 12 mo of life where it was observed that formula feeding was associated with a higher gain in fat mass and a fall in HMW adiponectin levels (7). In terms of health perspectives, we retrospectively analyzed
Table 1. Clinical and biochemical characteristics of obese children and adolescents according to group of neonatal feeding

\begin{tabular}{|c|c|c|c|}
\hline Variables & Group A & Group B & Group C \\
\hline $\operatorname{Sex}(M / F)$ & $26 / 15$ & $24 / 13$ & $19 / 16$ \\
\hline Age (y) & $10.6 \pm 0.5$ & $11.2 \pm 0.4^{*}$ & $10.6 \pm 0.4^{* *}$ \\
\hline Tanner 1 & $53.7 \%$ & $21.6 \%$ & $37.1 \%$ \\
\hline Tanner 2-3 & $19.5 \%$ & $40.5 \%$ & $40.0 \%$ \\
\hline Tanner 4-5 & $26.8 \%$ & $37.8 \%$ & $22.9 \%$ \\
\hline Breastfeeding (mo) & $15.2 \pm 1.2$ & $4.0 \pm 0.2^{*}$ & $0.02 \pm 0.06^{* *+}$ \\
\hline Time of weaning (mo) & $5.6 \pm 0.2$ & $5.7 \pm 0.2$ & $5.4 \pm 0.1$ \\
\hline Gestational age (wk) & $40.1 \pm 0.1$ & $39.3 \pm 0.1^{*}$ & $39.5 \pm 0.1^{* *}$ \\
\hline Neonatal weight $(\mathrm{kg})$ & $3.46 \pm 0.06$ & $3.42 \pm 0.09$ & $3.28 \pm 0.10^{* *}$ \\
\hline Neonatal length (cm) & $51.2 \pm 0.5$ & $50.6 \pm 0.4^{*}$ & $50.5 \pm 0.5^{* *}$ \\
\hline BMI $\left(\mathrm{kg} / \mathrm{m}^{2}\right)$ & $27.6 \pm 0.7$ & $27.9 \pm 0.7$ & $28.0 \pm 0.6$ \\
\hline BMISDS $\left(\mathrm{kg} / \mathrm{m}^{2}\right)$ & $2.067 \pm 0.088$ & $2.065 \pm 0.103$ & $2.052 \pm 0.078$ \\
\hline WC $(\mathrm{cm})$ & $91.1 \pm 2.3$ & $93.4 \pm 2.6$ & $90.2 \pm 1.6$ \\
\hline $\mathrm{SBP}(\mathrm{mmHg})$ & $127.0 \pm 2.1$ & $131.4 \pm 2.5$ & $128.6 \pm 2.7$ \\
\hline $\mathrm{DBP}(\mathrm{mmHg})$ & $82.0 \pm 1.6$ & $84.0 \pm 2.1$ & $86.1 \pm 2.1$ \\
\hline $\mathrm{T}-\mathrm{c}(\mathrm{mg} / \mathrm{dl})$ & $141.8 \pm 4.3$ & $137.1 \pm 3.4$ & $146.1 \pm 5.1$ \\
\hline $\mathrm{HDL}-\mathrm{c}(\mathrm{mg} / \mathrm{dl})$ & $38.5 \pm 1.3$ & $38.6 \pm 1.2$ & $40.8 \pm 1.5$ \\
\hline $\mathrm{TG}(\mathrm{mg} / \mathrm{dl})$ & $82.5 \pm 5.5$ & $80.4 \pm 6.3$ & $82.2 \pm 5.4$ \\
\hline $\mathrm{Glc} 0^{\prime}(\mathrm{mg} / \mathrm{dl})$ & $88.7 \pm 1.1$ & $87.8 \pm 1.2$ & $88.1 \pm 1.2$ \\
\hline Glc $120^{\prime}(\mathrm{mg} / \mathrm{dl})$ & $111.3 \pm 3.7$ & $113.6 \pm 2.8$ & $111.8 \pm 3.7$ \\
\hline Mean Glc (mg/dl) & $111.0 \pm 2.5$ & $114.6 \pm 2.3$ & $113.2 \pm 2.8$ \\
\hline Ins $0^{\prime}(\mathrm{mUI} / \mathrm{l})$ & $13.5 \pm 1.3$ & $17.8 \pm 2.4$ & $14.9 \pm 1.4$ \\
\hline Mean ins (mUI/l) & $67.0 \pm 6.1$ & $81.4 \pm 8.9$ & $67.3 \pm 7.0$ \\
\hline HOMA-IR & $3.0 \pm 0.3$ & $3.9 \pm 0.5$ & $3.2 \pm 0.3$ \\
\hline Total Adpn $(\mu \mathrm{g} / \mathrm{ml})$ & $6.8 \pm 0.6$ & $6.5 \pm 0.6$ & $7.6 \pm 0.9$ \\
\hline HMW Adpn ( $\mu \mathrm{g} / \mathrm{ml})$ & $3.6 \pm 0.4$ & $3.5 \pm 0.5$ & $4.1 \pm 0.6$ \\
\hline MMW Adpn $(\mu \mathrm{g} / \mathrm{ml})$ & $2.5 \pm 0.5$ & $2.9 \pm 0.7$ & $2.2 \pm 0.6$ \\
\hline M-weight pre-P (kg) & $60.4 \pm 2.4$ & $63.4 \pm 3.3$ & $65.0 \pm 2.3^{* *}$ \\
\hline $\mathrm{M}$-weight end-P $(\mathrm{kg})^{\mathrm{a}}$ & $76.1 \pm 3.2$ & $74.4 \pm 3.3$ & $83.6 \pm 3.1^{* *}$ \\
\hline M-BMI pre-P $\left(\mathrm{kg} / \mathrm{m}^{2}\right)^{\mathrm{a}}$ & $22.7 \pm 0.9$ & $23.7 \pm 0.1$ & $24.3 \pm 0.9$ \\
\hline M-BMl end-P $\left(\mathrm{kg} / \mathrm{m}^{2}\right)^{\mathrm{a}}$ & $28.5 \pm 1.2$ & $27.9 \pm 1.0$ & $31.3 \pm 1.1^{* *+}$ \\
\hline
\end{tabular}

Data are expressed as mean \pm SEM. Group A: prolonged breastfeeding ( $>6 \mathrm{mo})$. Group

B: short breastfeeding (1-6 mo). Group C: formula feeding from the birth.

Adpn, adiponectin; BMI, body mass index; BMISDS, body mass index standard

deviation scores; DBP, diastolic blood pressure; F, female; Glc O', fasting glucose; Glc

120', postchallenge glucose; $\mathrm{HDL}-\mathrm{C}, \mathrm{HDL}$ cholesterol; HMW, high molecular weight; HOMA-IR, homeostatic model assessment insulin resistance; Ins 0 ', fasting insulin; $\mathrm{M}$, male; M-BMI pre-and end-P, mothers' prepregnancy and at the end of pregnancy BMI; M-weight pre-and end-P, mothers' prepregnancy and at the end of pregnancy weight; MMW, medium molecular weight; SBP, systolic blood pressure; T-c, total cholesterol; TG, triglycerides; WC, waist circumference.

a Data available for 87 mothers. *Significance group A vs. B ( $P<0.05$ for age, gestational age, and neonatal length; $P<0.0001$ for breastfeeding). ${ }^{*}$ Significance group A vs. $C$ $(P<0.05$ for age, gestational age, and neonatal length; $P<0.03$ for neonatal weight and $M$-weight pre- $P ; P<0.02$ for $M$-weight and M-BMl end- $P ; P<0.0001$ for breastfeeding);

tSignificance group B vs. C ( $<0.02$ for M-BMI end-P; $P<0.0001$ for breastfeeding).

obese children born AGA with respect to breastfeeding and formula feeding in the first year of life. On the basis of this analysis, we failed to detect differences in total, HMW, and MMW adiponectin levels, as well as in clinical and biochemical 
a

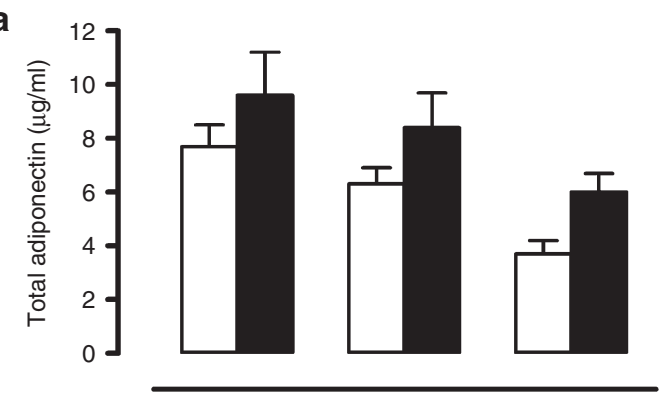

b

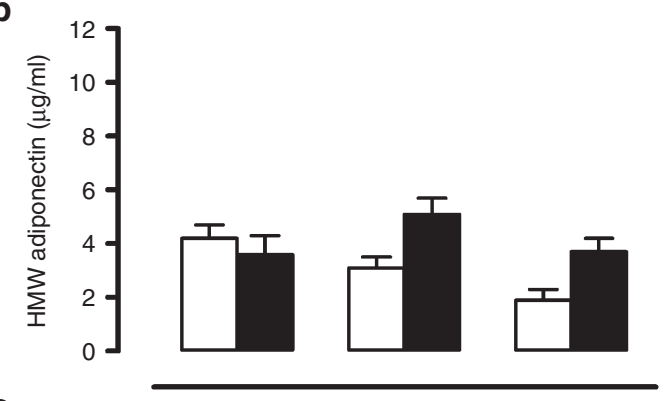

C

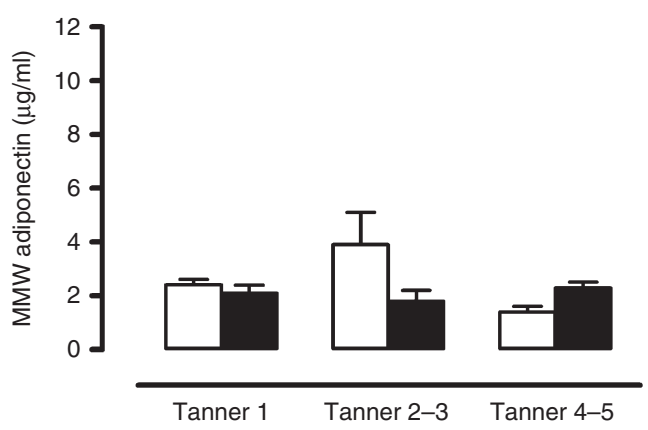

Figure 1. Serum adiponectin isoform levels with respect to sex and Tanner stage. Total (a), high-molecular weight (HMW) (b), and mediummolecular weight (MMW) (c) adiponectin $(\mu \mathrm{g} / \mathrm{ml})$ with respect to sex and Tanner stages. Data are expressed as mean \pm SEM. White bars: males. Black bars: females. Total and HMW adiponectin are higher in Tanner 1 males and females than in Tanner $4-5$ males $(P<0.005)$ and females $(P<0.01)$. Total and HMW adiponectin are also higher in Tanner $2-3$ than in Tanner 4-5 males $(P<0.05)$ and females $(P<0.04)$.

characteristics, even when breastfeeding was dichotomized into short- $(<6 \mathrm{mo})$ or long-term $(>6 \mathrm{mo})$ breastfeedings.

It has been postulated that feeding practice may modify or determine the long-term health risks. Adipose tissue is one of the fastest growing organs in early life $(1,3)$, and it has been shown that formula feeding can affect adiposity and that breastfeeding for at least $6 \mathrm{mo}$ can protect from obesity and cardiovascular diseases $(5,17)$; this is particularly true in preterm children $(5,18)$. Data on the future risk of obesity are mainly derived from studies on cohorts of adults, which suggest a U-shape relationship between human birth weight, obesity, and its comorbidities (1). On the other hand, children who are already obese and are born large for gestational age present a lower trunk fat percentage, higher adiponectin levels, a higher insulin sensitivity, a lower hepatic insulin resistance, and a lower insulin and free fatty acid concentration during OGTT, than their obese counterparts born SGA or AGA (19). To date, data on children born AGA and on the duration of breastfeeding are few and generally discordant. Several observational studies suggest that prolonged breastfeeding, in particular over 6 mo, reduces inflammation in children and adolescents $(5,20)$. On the other hand, a cluster randomized trial over $11.5 \mathrm{y}$ has recently demonstrated that an intervention to improve breastfeeding duration exclusively among healthy term infants did not influence a child's cardiometabolic risk factors, obesity prevalence, or alterations in hormones such as insulin-like growth factor I and adiponectin $(21,22)$. Our observational data are in agreement with this study, where in fact we failed to detect any difference in the distribution of adiponectin isoforms as well as in the metabolic characteristics of obese children born AGA with respect to the type of feeding and its duration in the first year. These results suggest that if obesity is already present in children who were born healthy and eutrophic, the protection from breastfeeding is overtaken by later factors, such as diet composition (23) or environment. In agreement with this concept, it has been shown that weight gain after $4 \mathrm{y}$ is one of the elements that mainly contributes to the deterioration of insulin resistance, also through a decrease in adiponectin secretion (19). Our data on maternal prepregnancy and at the end of pregnancy BMI are in line with the role of other factors beyond breastfeeding that could have an imprinting action on metabolism. In fact, we have shown that total and MMW adiponectin negatively correlate with prepregnancy and at the end of pregnancy BMI in obese female and male offspring, respectively. It has been widely demonstrated that pre and postpregnancy weight have a greater impact on neonatal adipokine distribution $(24,25)$. On the other hand, our data are of particular interest with respect to a gender dimorphism and are in line with similar data in neonate cord blood (26), suggesting that prepregnancy and gestational weight could have a long-term impact on metabolic risk. The reason as to why the isoforms are gender dimorphic is yet to be determined and will likely assist in understanding the physiology of adipose tissue programing and the risk of metabolic diseases in offspring.

Despite the results obtained, we cannot exclude that the protection exerted by a prolonged breastfeeding will become relevant at the end of adolescence, when the distribution and rearrangement of fat mass are completely determined. This hypothesis could justify some of the evidence presented for the adult populations. To prove that breastfeeding can be an independent regulator of total and HMW adiponectin, we will need to control for a number of confounding factors and use a more elaborate experimental design, being that a retrospective cross-sectional study is unable to demonstrate a causative role. Accordingly, adiponectin secretion is strictly influenced by gender, in particular the pediatric age. More precisely, a decrease in total and HMW adiponectin through puberty in males but not in females is negatively associated with androgen levels (27-30). In line with this, we have shown that total and HMW adiponectin concentrations are influenced by gender and Tanner stages. Interestingly, the correlation with BMISDS and waist was present only in males for HMW adiponectin, and total adiponectin did not correlate with BMISDS in females also when we corrected for confounders. 
Table 2. Correlation between total and HMW adiponectin and clinical and biochemical parameters according to sex

\begin{tabular}{|c|c|c|c|c|c|c|c|c|}
\hline & \multicolumn{4}{|c|}{ Total adiponectin } & \multicolumn{4}{|c|}{ HMW adiponectin } \\
\hline & \multicolumn{2}{|c|}{ Male } & \multicolumn{2}{|c|}{ Female } & \multicolumn{2}{|c|}{ Male } & \multicolumn{2}{|c|}{ Female } \\
\hline & $r$ & $P$ & $r$ & $P$ & $R$ & $P$ & $r$ & $P$ \\
\hline Breastfeeding & -0.133 & 0.289 & -0.025 & 0.880 & -0.112 & 0.374 & -0.018 & 0.916 \\
\hline Time of weaning & -0.153 & 0.231 & -0.018 & 0.909 & -0.142 & 0.266 & -0.059 & 0.719 \\
\hline Neonatal weight & -0.049 & 0.697 & -0.047 & 0.767 & -0.128 & 0.307 & -0.082 & 0.606 \\
\hline Neonatal length & -0.033 & 0.837 & -0.133 & 0.500 & 0.128 & 0.307 & 0.109 & 0.580 \\
\hline BMISDS & -0.239 & $<0.04$ & -0.143 & 0.354 & -0.296 & $<0.01$ & 0.008 & 0.960 \\
\hline WC & -0.360 & $<0.003$ & -0.301 & $<0.03$ & -0.295 & $<0.01$ & -0.116 & 0.459 \\
\hline SBP & -0.333 & $<0.006$ & 0.037 & 0.815 & -0.278 & $<0.02$ & -0.174 & 0.265 \\
\hline TG & -0.222 & 0.069 & -0.058 & 0.714 & -0.151 & 0.218 & -0.015 & 0.924 \\
\hline Glc $0^{\prime}$ & -0.057 & 0.639 & -0.201 & 0.089 & -0.014 & 0.910 & 0.049 & 0.756 \\
\hline Glc $120^{\prime}$ & -0.296 & $<0.02$ & -0.124 & 0.493 & -0.136 & 0.310 & -0.076 & 0.675 \\
\hline Mean Glc & -0.267 & $<0.04$ & -0.149 & 0.407 & -0.224 & 0.059 & -0.124 & 0.491 \\
\hline $\operatorname{lns} 0^{\prime}$ & -0.213 & 0.074 & -0.104 & 0.511 & -0.159 & 0.192 & 0.024 & 0.878 \\
\hline Mean ins & -0.309 & $<0.01$ & -0.320 & $<0.03$ & -0.192 & 0.142 & -0.128 & 0.479 \\
\hline HOMA-IR & -0.194 & 0.110 & -0.136 & 0.389 & -0.148 & 0.226 & 0.023 & 0.887 \\
\hline M-weight pre-Pa & -0.012 & 0.939 & -0.133 & 0.455 & -0.033 & 0.838 & -0.070 & 0.757 \\
\hline
\end{tabular}

Italics: significant values.

BMI, body mass index; BMISDS, body mass index standard deviation scores; DBP, diastolic blood pressure; Glc 0, fasting glucose; Glc 120; postchallenge glucose; HDL-C, HDL

cholesterol; HMW, high molecular weight; HOMA-IR, homeostatic model assessment insulin resistance; Ins 0 ', fasting insulin; M-BMI pre- and end-P, mothers' prepregnancy and at the end of pregnancy BMl; M-weight pre- and end-P, mothers' prepregnancy and at the end of pregnancy weight; T-c, total cholesterol; TG, triglycerides; $M$-weight pre and end-P, mother's pre-pregnancy and at the end of pregnancy weight and BMl; SBP, systolic blood pressure; WC, waist circumference.

a Data available for 87 mothers.

Similarly Andersen et al. (30) failed to observe a correlation between total adiponectin in prepubertal children whereas it was present in postpubertal adolescents, but very weakly in females with an $r$ of -0.19 , which is similar to our results (31). More recently, it has been shown that the influence of abdominal obesity influenced the adiponectin isoforms and that the impact of abdominal obesity was more evident in boys than in girls (32). Moreover, we observed that total and HMW adiponectin correlated with cardiometabolic risk factors in boys but not in girls, similarly to other papers on total adiponectin (33). All these findings suggest that the regulation of adiponectin, and as such the adipocyte environment, is very complex and is markedly regulated by changes with age and gender that are particularly evident in the pediatric phase.

In this study, we failed to detect any correlation between MMW adiponectin and biochemical or clinical parameters. Other papers have also failed to show a relationship between these isoforms and metabolic factors $(34,35)$. The role of MMW needs to be fully explored.
There are limitations in this study. First is the crosssectional design, in which we could not determine the causality of our results. Given the retrospective nature of the study, the second limitation was the inability to define precisely when children started to gain weight, as this window (before or after $4 \mathrm{y}$ of age) could have a role. Moreover, genetic or other environmental factors may also be important determinants.

In conclusion, this study demonstrates that adiponectin isoforms and metabolic characteristics are similarly distributed in obese children and adolescents who were born AGA, irrespective of the type and duration of feeding in the first year of life. Gender and gestational BMI are two of the most important contributors of adiponectin regulation in the pediatric age, and this should be always considered when adiponectin is under investigation. Further longitudinal studies are needed to understand whether breastfeeding, in those who were born eutrophic but developed obesity in childhood, protects from a metabolic impairment later in life. 


\section{MATERIALS AND METHODS Subjects}

This was a single-center cross-sectional retrospective study. Caucasian male and female children and adolescents with obesity were randomly recruited from those attending to the Pediatric Endocrine Service of our Hospital (May 2011-July 2013). Subjects were considered eligible if they were healthy, overweight or obese according to the International Obesity Task Force (IOTF) charts (36), diet-naive, born AGA from mothers who had a normal weight before pregnancy, and living in Novara surroundings in the first years, and if complete written anamnestic data of the first year of life and clinical and biochemical investigations inclusive of an OGTT at the first visit for obesity were available. Exclusion criteria were the known presence of type 1 diabetes, the use of drugs that influence glucose and lipid metabolism, blood pressure or appetite, and all causes of endocrine obesity. We also excluded subjects who underwent a prolonged hospitalization or chronically used pharmacological treatments in the first year of life. A written parental consent according to the principles of the Declaration of Helsinki was obtained prior to participation in the observational study. The study was approved by the Maggiore della Carità Hospital Ethical Committee (protocol 436/CE, study CE95/12; http://www.maggioreosp.novara.it).

\section{Clinical Evaluations}

Subjects underwent a complete clinical and evaluation at each visit by a research team experienced in pediatric endocrinology. Children were defined obese or overweight according to the IOTF charts (36). The pubertal stages were determined using the criteria and definitions described by Marshall and Tanner. Patients were divided into prepubertal (stage 1) and pubertal (stages 2-5) subjects. Height was measured by the Harpenden stadiometer and weight with light clothing by an electronic scale. BMI was calculated as body weight divided by squared height $\left(\mathrm{kg} / \mathrm{m}^{2}\right)$. Waist circumference was measured at the high point of the iliac crest around the abdomen and was recorded to the nearest $0.1 \mathrm{~cm}$. A nonelastic flexible tape measure was used. SBP and DBP were measured three times on the left arm and after $15 \mathrm{~min}$ at rest in the supine position using a standard mercury sphygmomanometer; the average was recorded and stratified according to pediatric percentiles of National High Blood Pressure Education Program Working Group on High Blood Pressure in Children and Adolescents (37).

The subjects were divided, according to feeding in the first year of life, into group A (prolonged breastfeeding, $>6$ mo), group B (short breastfeeding, 1-6 mo), and group $\mathrm{C}$ (formula feeding from birth or within the first $15 \mathrm{~d}$ after birth). Data on gestational age, birth weight, birth length, and weaning were also scheduled.

The weight conditions of mothers before and at the end of pregnancy were recorded on scheduled medical records. Data from 87 mothers were available.

\section{Biochemical Analysis}

After an overnight fasting, the blood samples for glucose, insulin, total cholesterol, high-density lipoprotein (HDL)-cholesterol, triglycerides, total, HMW, and MMW adiponectin were measured. Glucose and insulin were also measured every $30 \mathrm{~min}$ during OGTT (1.75 g of glucose solution $/ \mathrm{kg}$, maximum $75 \mathrm{~g}$ ).

The quantification of adiponectin multimeric complexes was performed with an enzyme-immunoassay kit (ALPCO Diagnostics, Salem, $\mathrm{NH}$ ). Serum levels of total, HMW, MMW, and LMW adiponectin were determined in duplicates from each subject and were diluted according to the manufacturer's instructions. This assay is able to quantify total, HMW + MMW, and HMW adiponectin directly. The concentrations of LMW and MMW are obtained by subtracting HMW + MMW from total adiponectin and HMW from HMW + MMW, respectively. The sensitivity of the assay is $19 \times 10^{-6} \mu \mathrm{g} / \mathrm{ml}$. As the assay quantifies directly total adiponectin, HMW and MMW collectively and the HMW indipendently, we decided to exclude the LMW values, which are not a direct measure but are derived by a mathematical subtraction. [All other assays have been previously described] (38).

\section{Statistical Analysis}

Categorical variables were expressed as frequencies or percentages. Distributions of continuous variables were examined for skewness and were logarithmically transformed where appropriate, with all the data expressed as mean \pm SEM to assist with interpretation. A sample of 24 individuals for each group was estimated to be sufficient to demonstrate a difference of $0.4 \mu \mathrm{g} / \mathrm{ml}$ in total and HMW adiponectin with an SD of 0.4 with $90 \%$ power and a significance level of $95 \%$, using the Student's $t$-test. The frequencies were compared using $\chi^{2}$ test or Fisher exact test, where appropriate. An analysis of covariance was used to determine the differences in subjects among groups. The covariates were age, gender, pubertal stage, BMISDS in model 1, the latter plus neonatal weight in model 2 or plus BMI of the mothers at the end of pregnancy in model 3. Correlations of total, HMW, and MMW adiponectin with continuous values of clinical and biochemical parameters were examined using Pearson correlation coefficients. A partial correlation was used to correct for covariates. Statistical significance was assumed for $P<0.05$. The statistical analysis was performed with SPSS for Windows V.17.0 (SPSS, Chicago, IL).

\section{ACKNOWLEDGMENTS}

The authors thank Cristina Fiorito, Giulia Genoni, and Alice Monzani for their technical assistance.

\section{STATEMENT OF FINANCIAL SUPPORT}

This study was supported by Regione Piemonte (Ricerca Finalizzata 2008, prot. n.2827), University of Piemonte Orientale, Novara, Italy.

Disclosure: The authors declare no conflict of interest. There is no conflict of interest that could be perceived as prejudicing the impartiality of the research reported.

\section{REFERENCES}

1. Desai M, Beall M, Ross MG. Developmental origins of obesity: programmed adipogenesis. Curr Diab Rep 2013;13:27-33.

2. Breton C. The hypothalamus-adipose axis is a key target of developmental programming by maternal nutritional manipulation. J Endocrinol 2013;216:R19-31.

3. Symonds ME, Mendez MA, Meltzer HM, et al. Early life nutritional programming of obesity: mother-child cohort studies. Ann Nutr Metab 2013;62:137-45.

4. Agostoni C, Braegger C, Decsi T, et al. Breast-feeding: a commentary by the ESPGHAN Committee on Nutrition. J Pediatr Gastroenterol Nutr 2009;49:112-25.

5. Fewtrell MS. Breast-feeding and later risk of CVD and obesity: evidence from randomised trials. Proc Nutr Soc 2011;70:472-7.

6. Guardamagna O, Abello F, Cagliero P, Lughetti L. Impact of nutrition since early life on cardiovascular prevention. Ital J Pediatr 2012;38:73.

7. de Zegher F, Sebastiani G, Diaz M, Gómez-Roig MD, López-Bermejo A, Ibáñez L. Breast-feeding vs formula-feeding for infants born small-forgestational-age: divergent effects on fat mass and on circulating IGF-I and high-molecular-weight adiponectin in late infancy. J Clin Endocrinol Metab 2013;98:1242-7.

8. de Zegher F, Sebastiani G, Diaz M, Sánchez-Infantes D, Lopez-Bermejo A, Ibáñez L. Body composition and circulating high-molecular-weight adiponectin and IGF-I in infants born small for gestational age: breast- versus formula-feeding. Diabetes 2012;61:1969-73.

9. Yamauchi T, Kadowaki T. Adiponectin receptor as a key player in healthy longevity and obesity-related diseases. Cell Metab 2013;17:185-96.

10. Hickman IJ, Whitehead JP. Structure, signalling and physiologic role of adiponectin-dietary and exercise- related variations. Curr Med Chem 2012;19:5427-43.

11. Scherer PE, Williams S, Fogliano M, Baldini G, Lodish HF. A novel serum protein similar to $\mathrm{C} 1 \mathrm{q}$, produced exclusively in adipocytes. J Biol Chem 1995;270:26746-9.

12. Shetty S, Kusminski CM, Scherer PE. Adiponectin in health and disease: evaluation of adiponectin-targeted drug development strategies. Trends Pharmacol Sci 2009;30:234-9.

13. Lara-Castro C, Luo N, Wallace P, Klein RL, Garvey WT. Adiponectin multimeric complexes and the metabolic syndrome trait cluster. Diabetes 2006;55:249-59. 
14. Kotani Y, Yokota I, Kitamura S, Matsuda J, Naito E, Kuroda Y. Plasma adiponectin levels in newborns are higher than those in adults and positively correlated with birth weight. Clin Endocrinol (Oxf) 2004;61:418-23.

15. Savino F, Fissore MF, Liguori SA, Oggero R. Can hormones contained in mothers' milk account for the beneficial effect of breast-feeding on obesity in children? Clin Endocrinol (Oxf) 2009;71:757-65.

16. Newburg DS, Woo JG, Morrow AL. Characteristics and potential functions of human milk adiponectin. J Pediatr 2010;156:Suppl 2:S41-6.

17. Andres A, Shankar K, Badger TM. Body fat mass of exclusively breastfed infants born to overweight mothers. J Acad Nutr Diet 2012;112:991-5.

18. Singhal A, Cole TJ, Lucas A. Early nutrition in preterm infants and later blood pressure: two cohorts after randomised trials. Lancet 2001;357:413-9.

19. Bouhours-Nouet N, Dufresne S, de Casson FB, et al. High birth weight and early postnatal weight gain protect obese children and adolescents from truncal adiposity and insulin resistance: metabolically healthy but obese subjects? Diabetes Care 2008;31:1031-6.

20. Labayen I, Ortega FB, Ruiz JR, et al. Association of exclusive breastfeeding duration and fibrinogen levels in childhood and adolescence: the European Youth Heart Study. Arch Pediatr Adolesc Med 2012;166:56-61.

21. Martin RM, Patel R, Kramer MS, et al. Effects of promoting longer-term and exclusive breastfeeding on adiposity and insulin-like growth factor-I at age 11.5 years: a randomized trial. JAMA 2013;309:1005-13.

22. Martin RM, Patel R, Kramer MS, et al. Effects of promoting longer-term and exclusive breastfeeding on cardiometabolic risk factors at age 11.5 years: a cluster-randomized, controlled trial. Circulation 2014;129:321-9.

23. Yeung EH, Appel LJ, Miller ER 3rd, Kao WH. The effects of macronutrient intake on total and high-molecular weight adiponectin: results from the OMNI-Heart trial. Obesity (Silver Spring) 2010;18:1632-7.

24. Laudes M, Oberhauser F, Bilkovski R, et al. Human fetal adiponectin and retinol-binding protein (RBP)-4 levels in relation to birth weight and maternal obesity. Exp Clin Endocrinol Diabetes 2009;117:146-9.

25. Shankar K, Kang P, Harrell A, et al. Maternal overweight programs insulin and adiponectin signaling in the offspring. Endocrinology 2010;151: 2577-89.

26. Simón-Muela I, Näf S, Ballesteros M, et al. Gender determines the actions of adiponectin multimers on fetal growth and adiposity. Am J Obstet Gynecol 2013;208:481.e1-7.
27. Böttner A, Kratzsch J, Müller G, et al. Gender differences of adiponectin levels develop during the progression of puberty and are related to serum androgen levels. J Clin Endocrinol Metab 2004;89:4053-61.

28. Woo JG, Dolan LM, Daniels SR, Goodman E, Martin LJ. Adolescent sex differences in adiponectin are conditional on pubertal development and adiposity. Obes Res 2005;13:2095-101.

29. Punthakee Z, Delvin EE, O'loughlin J, et al. Adiponectin, adiposity, and insulin resistance in children and adolescents. J Clin Endocrinol Metab 2006;91:2119-25.

30. Andersen KK, Frystyk J, Wolthers OD, Heuck C, Flyvbjerg A. Gender differences of oligomers and total adiponectin during puberty: a crosssectional study of 859 Danish school children. J Clin Endocrinol Metab 2007;92:1857-62.

31. Ong KK, Frystyk J, Flyvbjerg A, Petry CJ, Ness A, Dunger DB. Sexdiscordant associations with adiponectin levels and lipid profiles in children. Diabetes 2006;55:1337-41.

32. Ochiai $H$, Shirasawa $T$, Nishimura R, et al. Abdominal obesity and serum adiponectin complexes among population-based elementary school children in Japan: a cross-sectional study. BMC Pediatr 2014;14:81.

33. Kaser S, Tatarczyk T, Stadlmayr A, et al. Effect of obesity and insulin sensitivity on adiponectin isoform distribution. Eur J Clin Invest 2008;38: 827-34.

34. Kantartzis K, Staiger H, Machann J, et al. Adiponectin oligomers and ectopic fat in liver and skeletal muscle in humans. Obesity (Silver Spring) 2009;17:390-2.

35. Goto M, Goto A, Morita A, et al.; Saku Cohort Study Group. Low-molecular-weight adiponectin and high-molecular-weight adiponectin levels in relation to diabetes. Obesity (Silver Spring) 2014; 22:401-7.

36. Cole TJ, Lobstein T. Extended international (IOTF) body mass index cutoffs for thinness, overweight and obesity. Pediatr Obes 2012;7:284-94.

37. National High Blood Pressure Education Program Working Group on High Blood Pressure in Children and Adolescents. The fourth report on the diagnosis, evaluation and treatment of high blood pressure in children and adolescents. Pediatrics 2004;114:555-76.

38. Prodam F, Ricotti R, Genoni G, et al. Comparison of two classifications of metabolic syndrome in the pediatric population and the impact of cholesterol. J Endocrinol Invest 2013;36:466-73. 